\title{
Small intestinal submucosa extracellular matrix envelopes secure cardiovascular implantable devices and promote healthy tissue remodeling
}

\author{
Daniel Deegan ${ }^{1}$ and Jerome Riebman ${ }^{1}$ \\ ${ }^{1}$ Aziyo Biologics Inc.
}

July 27, 2021

\begin{abstract}
Introduction: Cardiac implantable electronic devices (CIEDs) induce a foreign body reaction and persistent inflammatory response that produces a fibrotic capsule around the implanted material. The study's aim was to investigate the ability of small intestinal submucosa (SIS) extracellular matrix (ECM) envelopes to mitigate fibrotic tissue formation and secure devices within healthy, vascularized tissue. Methods: Clinically relevant pacemakers without leads were subcutaneously implanted in a pre-clinical rabbit model. Eleven CIEDs were placed in CanGaroo ECM Envelopes, while ten devices were implanted without envelopes and analyzed at 13 and 26 weeks. The subcutaneous pocket and newly formed connective tissue adjacent to the CIED were evaluated by an independent, blinded pathologist for the local tissue response and constructive remodeling. CIED movement in the pocket was also documented as implant sites were prone to abrasion due to normal rabbit behavior. Results: CanGaroo Envelopes surrounding the CIEDs remodeled into native tissue with normally organized collagen. The tissue had a lower average thickness at 13 and 26 weeks compared to tissue encapsulating CIEDs without envelopes. The tissue also scored significantly higher in neovascularization versus capsule tissue surrounding CIEDs without envelopes at 26 weeks. Use of the ECM envelope reduced the incidence of device flipping within the pocket by $40 \%$ compared to CIEDs without envelopes, showing the ability of CanGaroo Envelopes to secure CIEDs in place. Conclusions: CanGaroo ECM Envelopes remodeled into native, vascularized tissue surrounding clinically applicable CIEDs. This thinner, healthy tissue pocket secured CIEDs and significantly improved stabilization versus devices without envelopes over 26 weeks.
\end{abstract}

Title: Small intestinal submucosa extracellular matrix envelopes secure cardiovascular implantable devices and promote healthy tissue remodeling

Authors:

Daniel B. Deegan ${ }^{1}$

Jerome B. Riebman ${ }^{1}$

Institution Afilliations:

${ }^{1}$ Aziyo Biologics, Inc., Silver Spring, MD

Conflict of Interest Statement:

Daniel B. Deegan and Jerome B. Riebman are employees of Aziyo Biologics.

\section{Source of Funding Statement:}

This work was supported by Aziyo Biologics, Inc.

Corresponding Author Information: 
Daniel B. Deegan, Aziyo Biologics Inc., ddeegan@aziyo.com

\section{Key Words:}

\section{CIED; Extracellular matrix; CanGaroo envelope; device stabilization; tissue remodeling}

\section{Small intestinal submucosa extracellular matrix envelopes secure cardiovascular implantable devices and promote healthy tissue remodeling}

\section{Structured Abstract:}

Introduction: Cardiac implantable electronic devices (CIEDs) induce a foreign body reaction and persistent inflammatory response that produces a fibrotic capsule around the implanted material. The study's aim was to investigate the ability of small intestinal submucosa (SIS) extracellular matrix (ECM) envelopes to mitigate fibrotic tissue formation and secure devices within healthy, vascularized tissue.

Methods: Clinically relevant pacemakers without leads were subcutaneously implanted in a pre-clinical rabbit model. Eleven CIEDs were placed in CanGaroo ECM Envelopes, while ten devices were implanted without envelopes and analyzed at 13 and 26 weeks. The subcutaneous pocket and newly formed connective tissue adjacent to the CIED were evaluated by an independent, blinded pathologist for the local tissue response and constructive remodeling. CIED movement in the pocket was also documented as implant sites were prone to abrasion due to normal rabbit behavior.

Results: CanGaroo Envelopes surrounding the CIEDs remodeled into native tissue with normally organized collagen. The tissue had a lower average thickness at 13 and 26 weeks compared to tissue encapsulating CIEDs without envelopes. The tissue also scored significantly higher in neovascularization versus capsule tissue surrounding CIEDs without envelopes at 26 weeks. Use of the ECM envelope reduced the incidence of device flipping within the pocket by $40 \%$ compared to CIEDs without envelopes, showing the ability of CanGaroo Envelopes to secure CIEDs in place.

Conclusions: CanGaroo ECM Envelopes remodeled into native, vascularized tissue surrounding clinically applicable CIEDs. This thinner, healthy tissue pocket secured CIEDs and significantly improved stabilization versus devices without envelopes over 26 weeks.

\section{Introduction:}

Cardiac arrhythmias are often treated by use of cardiac implantable electronic devices (CIEDs), which include pacemakers, implantable cardioverter-defibrillators, and devices for cardiac resynchronization therapy. As examined in a variety of studies, these CIEDs evoke a foreign body reaction and prolonged inflammatory response in the subcutaneous tissue that stimulate the formation of a fibrotic tissue capsule surrounding the implanted material [1-5]. In high risk patients, CIED-induced scar tissue formation and incomplete surgical healing often result in complications at the implant sites of CIED generators or leads, such as migration, erosion, dislodgement, or infection, which require additional procedures and dissection of the device pockets [6-9]. In addition, younger patients are receiving CIEDs and surviving long enough to require multiple pulse generator change-outs and lead revisions $[10,11]$. Complication rates after generator or lead exchanges and upgrade or revision surgeries are considerable, and complication rates increase with each additional reoperative procedure [7, 11-15]. The thickness of the fibrotic tissue surrounding devices proportionately increases the difficulty of these procedures, while a lack of vascularization in the scar tissue greatly elevates the risks of post-surgery infection $[4,12,16]$. A product that mitigates the local tissue response to CIEDs and promotes healthy, vascularized tissue formation around the devices can improve patient outcomes and reduce complications of the implant or revision surgeries.

To address the difficulties caused by patients' immune reactions to CIEDs and neurostimulator devices, a natural extracellular matrix (ECM) envelope product called CanGaroo (Aziyo Biologics) was developed to wrap electronic implants. Biologic ECM scaffolds are created from various processes and tissue sources to promote healthy, site-specific tissue remodeling in patients [17]. CanGaroo utilizes non-chemically crosslinked multilaminate sheets of decellularized, lyophilized porcine small intestinal submucosa (SIS) that are strong 
and supportive in structure, but compliant and porous enough to allow cell infiltration and achieve optimal tissue remodeling outcomes [18-20].

SIS is an ECM biomaterial that has been well-characterized in multiple regenerative medicine applications [18]. SIS ECM is rich in growth factors, such as basic fibroblast growth factor (bFGF) and vascular endothelial growth factor (VEGF), as well as glycosaminoglycans (GAGs) and structural proteins, such as heparin sulfate, collagens, and fibronectin [21,22]. These natural ECM structures and bioactive factors, absent in synthetic scaffolds, effectively reduce dense fibrous scarring by stimulating healthy tissue regeneration and angiogenesis [23-25]. This capacity is at least partially attributed to the fact that SIS promotes macrophages with a pro-remodeling, anti-inflammatory M2 phenotype versus a pro-inflammatory M1 phenotype [26]. When placed around an implanted electronic device, the SIS ECM envelope naturally resorbs over time and releases intrinsic biologic signals which mitigate fibrotic encapsulation normally caused by an inflammatory response to the foreign generator [27]. By supporting host cell infiltration and proliferation and promoting anti-inflammatory immune cell phenotypes such as M2 macrophages, SIS ECM envelopes create a conducive environment for constructive remodeling [28, 29].

In this study using a clinically relevant subcutaneous rabbit model, we tested the ability of CanGaroo ECM Envelopes to mitigate fibrotic tissue and scar formation induced by implantation of CIEDs for up to 26 weeks. The study also analyzed the natural resorption and remodeling of the SIS ECM envelopes over time, while evaluating neovascularization, normal collagen organization, and necrosis. Finally, by using full-size, clinically relevant CIEDs, we observed the ability of CanGaroo Envelopes to stabilize the devices in healthy tissue within the subcutaneous pockets of a model highly susceptible to device movement due to normal rabbit motion and behavior.

\section{Methods:}

Animal ModelA subcutaneous implantation study was designed in New Zealand White (NZW) rabbits using dorsal bilateral surgeries to implant CIEDs with or without supportive CanGaroo ECM Envelopes. The New Zealand White rabbit was chosen because it provided enough surface area on the dorsal surface for implantation of the test and control articles of a clinically relevant size (pacemakers approximately $5.5 \times 5.5$ $\mathrm{x} 0.5 \mathrm{~cm}$ ). The rabbit is an appropriate subcutaneous pre-clinical model for evaluating biocompatibility and local effects of implanted materials according to the current ISO testing standards (ISO 10993-6) because of their competent immune system and skin thickness similar to that of humans, as opposed to other small animal models such as rats and mice.

Study Design The study protocol was approved by the Institutional Animal Care and Use Committee (IACUC) and all animals received humane care in compliance with "The Guide for the Care and Use of Laboratory Animals," published by the US National Institutes of Health. Twenty one (21) NZW rabbits were implanted with CIEDs with eleven being placed in CanGaroo ECM Envelopes. Midline incisions were created on the dorsal lumbar aspect of each animal, per veterinary practice. Subcutaneous pockets were created with minimal pocket dissection and just large enough to accommodate one implant per pocket.

For the experimental group, eleven single-chamber pacemakers (St. Jude Medical) without leads were placed in CanGaroo Envelopes (Size Medium, Aziyo Biologics, Lot\# M17E1116) (Figure 1). The pacemaker lead header was oriented nearest to the opening in the hydrated envelope, and the lead header was placed in the implant pocket closest to the skin incision. The envelope-CIED assembly was secured with a single, nonabsorbable suture (3-0 Prolene) that passed across the envelope opening edges in order to prevent incidental release of the CIED and secured to the underlying chest wall. A second non-absorbable suture was passed through the chest wall, envelope, and pacemaker header suture hole to secure the implant. For the control group, ten CIEDs without envelopes were again oriented with the lead header closest to the skin incision and secured in place through the suture hole with a single, non-absorbable suture. The pocket surrounding each implant was closed to contain the implant using a continuous stitch and non-absorbable suture to reconnect the subcutaneous tissues around each implant. From there, standard surgical techniques were used to finish closing the implant sites. 

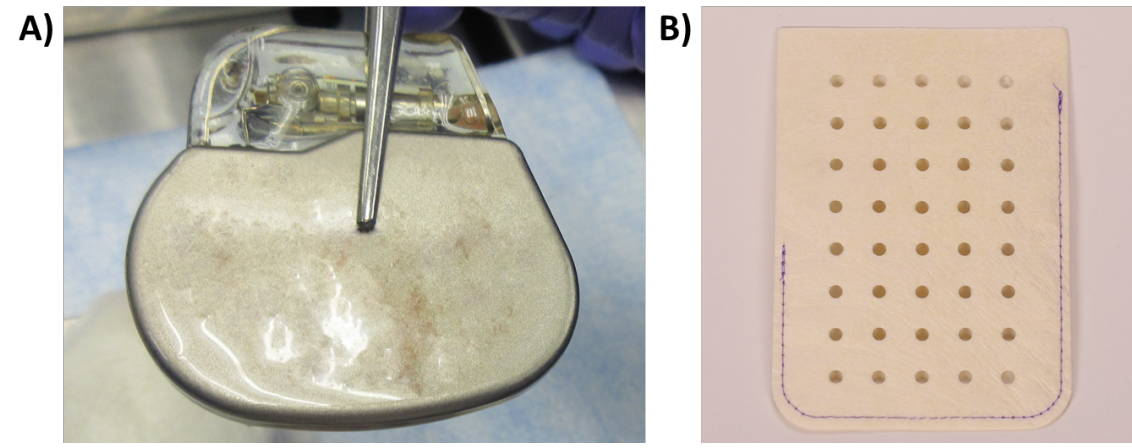

Figure 1: Images of test and control articles- A) The image shows the clinically relevant CIED model used for the surgical implants. B) The experimental group enclosed CIEDs in a CanGaroo ECM Envelope like the one pictured here.

Upon closing the incision sites, the animals were allowed to recover and were returned to standard individual housing. The incision sites were continually monitored over the course of the study for any abnormalities or incomplete wound healing. Animals were individually housed in stainless steel cages/polycarbonate cages with slatted floors in environmental conditions of temperature, relative humidity, and photo-period according to NIH recommendations for this species as reported in the "Guide for the Care and Use of Laboratory Animals". Potable water, certified commercial feed, and environmental enrichment were supplied until animals were sacrificed at scheduled time points.

One animal receiving a CIED with CanGaroo ECM Envelope was sacrificed and evaluated at 2 weeks post implant (the time point for skin incision healing) to provide a histological starting point for expected ECM envelope resorption and remodeling, which is designed to occur naturally over time in the pocket. For the remaining animals in each group, four implants were evaluated at week 13, and six were evaluated at 26 weeks. At each time point, rabbits were humanely euthanized. Implant sites were then opened, observed macroscopically, and then processed for histopathology.

For macroscopic observations, the outer capsule surrounding the CIED at each implant site was surgically exposed and photographed by removing the layers of epidermis and dermis. Evidence of normal ECM envelope resorption and constructive remodeling was documented. Presence of fluid and capsule thickening or discoloration were also noted. Before and upon capsule dissection, the surgeon recorded any movement or flipping of the CIED from its original implanted position. The CIED was then removed, and the full capsule was excised and marked with tissue dye for orientation. The tissue was fixed in formalin for histopathological analysis.

Histopathology and Capsule Measurements Two (2) tissue pieces 1cm in length were excised for histology from the middle and dorsal corner of each capsule. Samples were processed by standard histopathology techniques including paraffin embedding, sectioning (two serial sections at each sample midpoint), and staining (one section per stain with hematoxylin and eosin $[\mathrm{H} \& \mathrm{E}]$ and Trichrome). Slides were evaluated by a blinded veterinary pathologist for local tissue responses, envelope resorption, and fibrotic tissue formation. This analysis scored the appearance of mineralization, necrosis, neovascularization, and fibrosis on an established scale of 0 to 4 ( 0 meaning absent and 4 meaning marked presence).

For assessment of CanGaroo ECM Envelope resorption which directly correlates with remodeling, a baseline estimation of envelope area was made in the slides from an initial 2 week time point. Subsequent measurements of remnant product in the capsular tissue were made at 13 and 26 weeks and standardized to the 2 week time point. In addition to resorption, thickness measurements of the tissue capsule were made at three representative places from each individual $\mathrm{H} \& \mathrm{E}$ and trichrome slide of each tissue sample, excluding remnant envelope material in the measurements. The average of these measurements was used to estimate fibrotic capsule thickness from every individual implant site and subsequently averaged for each experimental group. 


\section{Results: Gross Necropsy Observations :}

Upon examination of the subcutaneous pocket, normal, mild amounts of fluid from surgical wound healing were present in both implant groups at 13 weeks. Fluid dissipated and became absent by the week 26 time point. Connective tissue bands, associated with a normal subcutaneous response to implants, were observed in both groups at the 13 and 26 week time points with the tissue thickness increasing with implant duration. Compared to intact envelope material and related tissue discoloration found in the CIED with CanGaroo ECM Envelope group at 2 weeks, clear and steady progress was seen in the tissue remodeling of the envelope by 13 and 26 weeks. This remodeling was accompanied by an increased level of vascularization over time that appeared greater than levels found in tissue surrounding the CIED without envelope group at 13 and 26 weeks(Figure 2) .

This connective tissue secured cardiac devices in place in the subcutaneous pocket. However, CIEDs were highly prone to movement within the pocket because of the normal, instinctive movement of the rabbits, including rolling onto the implant site or directly rubbing their dorsal side on the cage. Also, CIED leads were absent in this model, which usually restrict movement in the subcutaneous space and further stabilize the implants. This vulnerability lead to a high incidence of device flipping within the pocket. During necropsy, $50 \%$ or five (5) out of 10 total implants were documented to flip $180^{\circ}$ cranially within the pocket in the absence of an envelope. Only a single incidence of flipping out of 10 implants was observed at either the 13 or 26 week end points in the presence of CanGaroo ECM Envelopes.
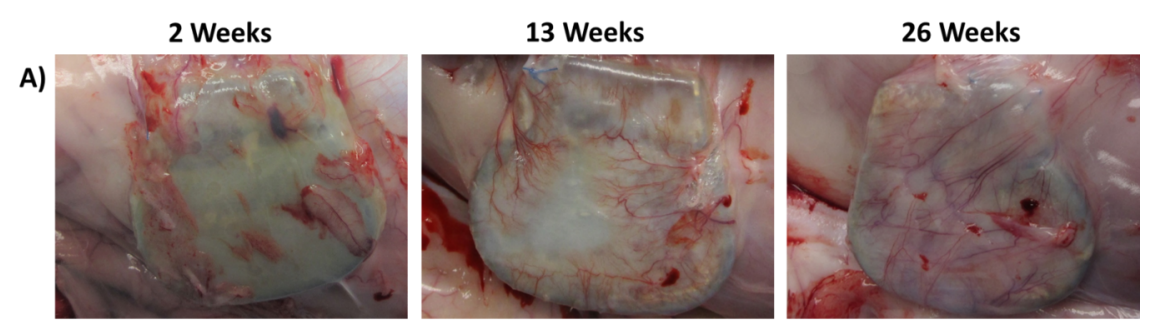

B)
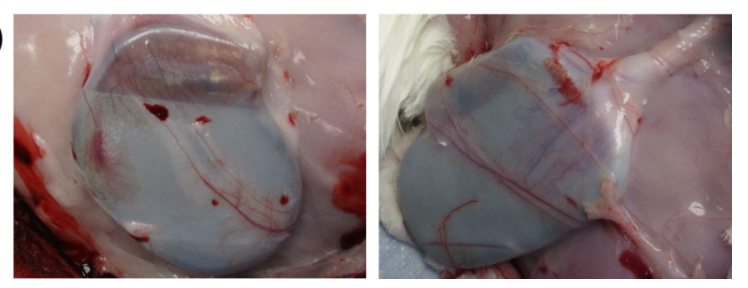

Figure 2: Representative gross images of the subcutaneous pocket- A) Images show remodeling of the ECM envelope over time at 2, 13, and 26 weeks with increasing levels of vascularization. B) Comparison images from the CIED without envelope implant sites at 13 and 26 weeks show similar thickening of connective tissue but lower vascularization levels and a flipped device at 26 weeks.

\section{Histopathology:}

Histology was performed on the capsular tissue of the CIED with CanGaroo ECM Envelope and CIED without envelope groups to quantify and score the tissue response. H\&E and trichrome slides showed normal collagen formation and organization in the connective tissue, which was similar between the groups (Figure 3 ). The use of CanGaroo ECM Envelopes generated a lower average capsule thickness compared to CIEDs without envelopes with a mean of $349 \pm 165 \mu \mathrm{m}$ (mean $\pm \mathrm{SD}$ ) versus $417 \pm 152 \mu \mathrm{m}$ at 13 weeks and a mean of $427 \pm 141 \mu \mathrm{m}$ versus $439 \pm 91 \mu \mathrm{m}$ at 26 weeks. High standard deviation was seen in these measurements due to animal-to-animal variation in capsule formation. 

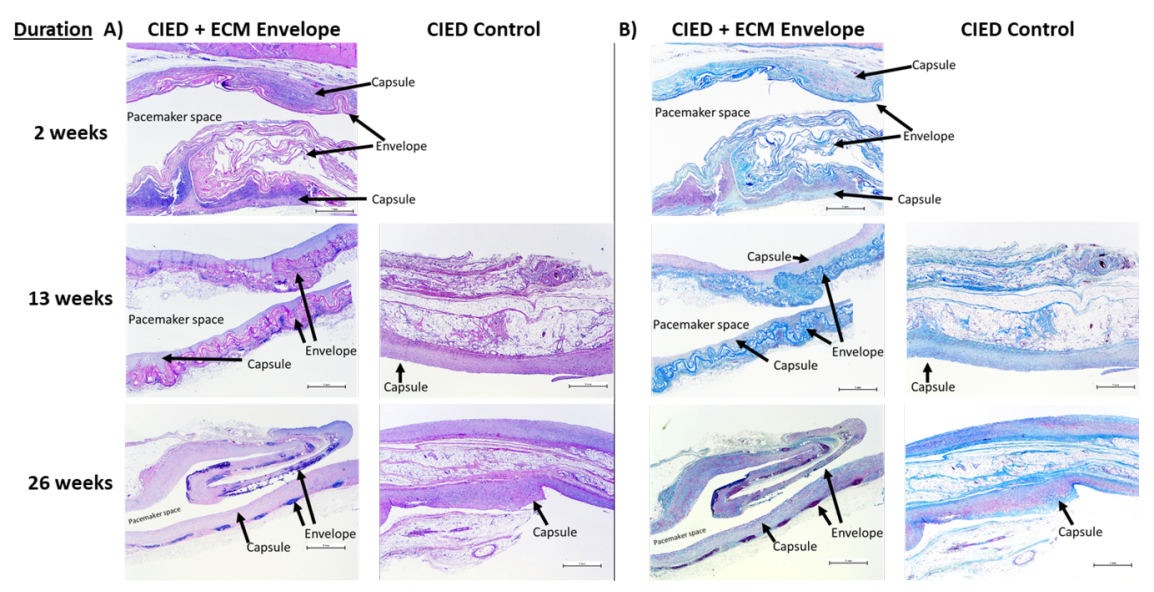

Figure 3: Representative A) H\&E and B) trichrome images of capsular tissue-Images show envelope resorption and capsule thickening over 2, 13 and 26 weeks in the presence of the ECM envelope. Images from pockets receiving CIEDs without envelopes showed similar connective tissue formation at 13 and 26 weeks.

Histology sections were also scored for neovascularization on a 0 to 4 point scale by an independent blinded pathologist (Table 1). The CanGaroo ECM Envelope group had a significantly higher neovascularization average score of 2.1 compared to a mean of 1.7 in the CIED without envelope group ( $p<.05$, Student's $t$-test) . In either group, no significant mineralization was observed in the tissue. No necrotic tissue areas were observed, and only low levels of apoptotic cells normally seen in surgical sites were found.

Table 1: Neovascularization scoring criteria

\begin{tabular}{|c|c|c|c|c|c|}
\hline $\begin{array}{l}\text { Tissue } \\
\text { Response }\end{array}$ & Score & Score & Score & Score & Score \\
\hline & $0=$ Absent & $1=$ Minimal & $2=$ Mild & $\begin{array}{l}3= \\
\text { Moderate }\end{array}$ & $4=$ Marked \\
\hline Neovascularization & Absent & $\begin{array}{l}\text { Minimal } \\
\text { capillary } \\
\text { proliferation (1-3 } \\
\text { capillary buds, } \\
\text { small blood } \\
\text { vessels, venules, } \\
\text { and / or } \\
\text { arterioles) }\end{array}$ & $\begin{array}{l}\text { Groups of } 4-7 \\
\text { capillaries }\end{array}$ & $\begin{array}{l}\text { Broad band of } \\
\text { capillaries with } \\
\text { supporting } \\
\text { structures }\end{array}$ & $\begin{array}{l}\text { Extensive band } \\
\text { of capillaries } \\
\text { with supporting } \\
\text { fibroblastic } \\
\text { structures }\end{array}$ \\
\hline
\end{tabular}

For the CIED with CanGaroo Envelope group, envelope resorption was quantified in histology slides as the average area of remnant material at 13 and 26 weeks standardized to material present at 2 weeks. By 26 weeks, $73.2 \%$ of the envelope material was resorbed and remodeled leaving $26.8 \%$ of the starting material (Table 2) .

Table 2: Comparison of the average area of implanted material within the capsular tissue slides taken from CanGaroo Envelope implant sites at 2, 13, and 26 week duration

\begin{tabular}{llll}
\hline Duration & $\mathbf{2}$ Weeks & $\mathbf{1 3}$ Weeks & 26 Weeks \\
\hline Average Area of Implanted Material within the Implant Sites $\left(\mathrm{mm}^{2}\right)$ & 14.52 & 5.72 & 3.89
\end{tabular}




\begin{tabular}{|c|c|c|c|}
\hline Duration & 2 Weeks & 13 Weeks & 26 Weeks \\
\hline Percent Change from 2 Weeks & & $60.6 \%$ & $73.2 \%$ \\
\hline
\end{tabular}

\section{Discussion:}

Presented with the challenges and complications associated with subcutaneous device implantations, this study demonstrated the ability of CanGaroo ECM Envelopes to secure clinically relevant CIEDs and mitigate fibrotic tissue capsule thickness in a pre-clinical animal model. The rabbit model that was used was highly susceptible to device movement in the pocket due to the range of motion of the animals and ability to directly aggravate the surgical site through rolling or rubbing. Devices also did not have leads attached, which usually limit the mobility of CIEDs. Despite these vulnerabilities, SIS ECM envelopes securing the CIEDs substantially reduced the incidence of device flipping by 5 times compared to the group containing CIEDs without envelopes. Device flipping could cause lead dislodgement in clinical patients [30]. This type of movement also creates friction and abrasion, which in more long term models or clinical cases could develop into CIED erosions and infections or the devices migrating outside of the subcutaneous pocket [8]. It should be noted that no erosions were observed in the study, as compared to a previous study utilizing a thin-skinned mouse model and customized biotelemetry devices [31].

In addition to stabilizing CIEDs in the study, use of SIS ECM envelopes correlated with a general reduction in thickness of the fibrotic capsule. However, capsule thickness varied greatly between different animals leading to high standard deviation in the data. Future studies could draw out significance by increasing animal numbers or screening animals to better standardize the immune system being challenged. SIS ECM envelopes mitigate the foreign body response to reduce fibrosis and promote a favorable environment for constructive tissue remodeling. CanGaroo is composed of non-crosslinked SIS ECM that elicits M2 macrophage polarization and production of IL-10 and other anti-inflammatory cytokines [24, 26, 28, 29, 32-34]. This ability is in direct contrast to synthetic scaffolds, which often favor a pro-inflammatory M1 macrophage phenotype, especially when the implanted material is non-degradable [24, 26].

SIS scaffolds contain a natural three-dimensional architecture that allows cells to migrate throughout the matrix. Fibronectin, laminin, and proteoglycans supply attachment sites to enable cells to remodel and organize new tissue [18]. GAGs of the proteoglycans also bind and protect important growth factors that are utilized in a controlled, time dependent manner by cells. Several of these growth factors in SIS promote scarless healing at the site of application, especially bFGF. bFGF accelerates wound healing and regulates organized collagen deposition by fibroblasts [35]. SIS recruits a variety of other cells types important for sustained tissue remodeling including endothelial cells, epithelial cells, and mesenchymal stem cells [36]. Cells recruited and signaled during the natural remodeling of the scaffold promote tissue regeneration, compared to cases of synthetic materials that often stimulate excess fibrosis or formation of poorly organized connective tissue [18].

Besides mitigating fibrosis, treatment with SIS ECM envelopes also improved angiogenesis and neovascularization. Using a 0 to 4 scoring criteria where a greater score represented more capillary proliferation, subcutaneous tissue surrounding CIEDs with CanGaroo Envelopes scored significantly higher than tissue adjacent to CIEDs without envelopes. This result is at least partially due to SIS's growth factor milieu of angiogenic growth factors such as TGF- $\beta, \mathrm{bFGF}$, and VEGF. These proteins are not only anti-inflammatory but promote angiogenesis by upregulating endothelial cell migration and proliferation $[18,21,37,38]$. Cells also mediate the proteolytic cleavage and release of matricryptic peptides from the scaffold's ECM, which have similar angiogenic effects on both endothelial cells and perivascular stem cells [39, 40]. The natural porosity of SIS ECM scaffolds supports cell infiltration and ultimately allows for fast and efficient vessel and capillary growth [18]. Generating healthy, vascularized tissue around the device facilitates easier and successful CIED change-outs and lowers the incidence of infection, which may be particularly important in a younger patient who will likely experience multiple change-outs $[11,12]$. These angiogenic advantages also 
translate to positive surgical outcomes in a variety of other clinical cardiovascular applications of SIS such as pericardial closure and cardiac and vascular reconstruction [41, 42].

Properly vascularized tissue grants the body's immune and regenerative cells direct access into the CIED pocket to aid in the steady resorption and remodeling of the CanGaroo Envelope. This process not only releases peptides from the SIS ECM with angiogenic potential, but the peptides also contain inherent antimicrobial properties, thereby assisting in the prevention of bacterial infections of the subcutaneous pocket $[18,38,43-45]$. Many synthetic or crosslinked scaffolds are non-degradable or can take years or more to fully resorb [24, 26]. In this rabbit study, CanGaroo Envelopes showed steady resorption and remodeling over time with about $26.8 \%$ of the starting material remaining after 26 weeks. The subcutaneous space in rabbits is slower to remodel biomaterials than humans, but full turnover of the remaining ECM would have been achieved soon thereafter. Although this timetable may be different from clinical cases, the ability of the host tissue to utilize and remodel the SIS ECM envelope into native tissue is conserved.

\section{Conclusions:}

This pre-clinical study demonstrated several advantages of using SIS ECM envelopes to wrap clinically relevant CIEDs during subcutaneous implantation. Compared to CIEDs alone without envelopes, procedures utilizing CanGaroo Envelopes promoted formation of thinner connective tissue bands and stimulated significantly higher rates of neovascularization, while avoiding tissue necrosis. The study supported the hypothesis that the CanGaroo ECM Envelopes steadily remodel and are replaced by native, organized collagen over time that is free of mineralization. This optimized tissue regeneration contributed to a five-fold reduction in CIED flipping within the subcutaneous pocket through stabilization by CanGaroo Envelopes. This study bolsters and compliments existing in vivo and clinical data that show CanGaroo Envelopes provide a superior pro-remodeling microenvironment for creation of healthy and fully vascularized tissue, which safely secures devices in place and improves the outcomes of device upgrades or revision surgeries.

\section{References:}

1. Anderson, J.M., A. Rodriguez, and D.T. Chang, Foreign body reaction to biomaterials. Seminars in immunology, 2008. 20 (2): p. 86-100.

2. Steckiewicz, R., et al., Morphometric parameters of cardiac implantable electronic device (CIED) pocket walls observed on device replacement. Folia Morphol, 2017. 76 (4): p. 675-681-675-681.

3. Perry, L., et al., Explanted Pacemakers: Observations of the Long-term Foreign Body Response. Journal of Undergraduate Research in Bioengineering, 2007(7): p. 13-21.

4. Maytin, M., R.M. John, and L.M. Epstein, Device Pocket Scar Predicts Transvenous Lead Extraction Difficulty. Journal of Innovations in Cardiac Rhythm Management, 2015. 6 (11): p. 2173-2177.

5. Biefer, H.R., et al., Generator pocket adhesions of cardiac leads: classification and correlation with transvenous lead extraction results. Pacing Clin Electrophysiol, 2013. 36 (9): p. 1111-6.

6. Kusumoto, F.M., et al., 2017 HRS expert consensus statement on cardiovascular implantable electronic device lead management and extraction. Heart Rhythm, 2017. 14 (12): p. e503-e551.

7. Borleffs, C.J., et al., Recurrent implantable cardioverter-defibrillator replacement is associated with an increasing risk of pocket-related complications. Pacing Clin Electrophysiol, 2010.33 (8): p. 1013-9.

8. Baddour, L.M., et al., Update on cardiovascular implantable electronic device infections and their management: a scientific statement from the American Heart Association. Circulation, 2010.121 (3): p. 458-77.

9. Cleland, J.G., et al., The effect of cardiac resynchronization on morbidity and mortality in heart failure. N Engl J Med, 2005.352 (15): p. 1539-49.

10. Beck, H., et al., 50th Anniversary of the first successful permanent pacemaker implantation in the United States: historical review and future directions. Am J Cardiol, 2010. 106 (6): p. 810-8. 
11. Tarakji, K.G., et al., Cardiac Implantable Electronic Device Infection in Patients at Risk. Arrhythm Electrophysiol Rev, 2016.5 (1): p. 65-71.

12. Robotti, F., et al., Microengineered biosynthesized cellulose as anti-fibrotic in vivo protection for cardiac implantable electronic devices. Biomaterials, 2020. 229 : p. 119583.

13. Polyzos, K.A., A.A. Konstantelias, and M.E. Falagas, Risk factors for cardiac implantable electronic device infection: a systematic review and meta-analysis. Europace, 2015. 17 (5): p. 767-77.

14. Poole, J.E., et al., Complication rates associated with pacemaker or implantable cardioverter-defibrillator generator replacements and upgrade procedures: results from the REPLACE registry.Circulation, 2010. 122 (16): p. 1553-61.

15. Olsen, T., et al., Incidence of device-related infection in 97750 patients: clinical data from the complete Danish device-cohort (1982-2018). Eur Heart J, 2019. 40 (23): p. 1862-1869.

16. Wilkoff, B.L., Infections associated with cardiac implantable electronic devices are misunderstood. Texas Heart Institute Journal, 2011. 38 (4): p. 353-354.

17. Cornwell, K.G., A. Landsman, and K.S. James, Extracellular matrix biomaterials for soft tissue repair. Clin Podiatr Med Surg, 2009. 26 (4): p. 507-23.

18. Brown-Etris, M., W. Cutshall, and M. Hiles, A New Biomaterial Derived from Small Intestine Submucosa and Developed into a Wound Matrix Device. Wounds, 2002. 14 (4): p. 150-166.

19. Sohail, M.R., et al., Preclinical evaluation of efficacy and pharmacokinetics of gentamicin containing extracellular-matrix envelope. Pacing Clin Electrophysiol, 2020. 43 (3): p. 341-349.

20. Brown, B.N. and S.F. Badylak, Extracellular matrix as an inductive scaffold for functional tissue reconstruction. Transl Res, 2014. 163 (4): p. 268-85.

21. Hodde, J.P., et al., Vascular endothelial growth factor in porcine-derived extracellular matrix. Endothelium, 2001. 8 (1): p. 11-24.

22. Badylak, S.F., D.O. Freytes, and T.W. Gilbert, Extracellular matrix as a biological scaffold material: Structure and function. Acta Biomaterialia, 2009. 5 (1): p. 1-13.

23. Piterina, A.V., et al., ECM-based materials in cardiovascular applications: Inherent healing potential and augmentation of native regenerative processes. Int J Mol Sci, 2009. 10 (10): p. 4375-417.

24. Brown, B.N., B.M. Sicari, and S.F. Badylak, Rethinking regenerative medicine: a macrophage-centered approach. Frontiers in immunology, 2014. 5 : p. 510-510.

25. Lin, X., et al., Small intestinal submucosa-derived extracellular matrix bioscaffold significantly enhances angiogenic factor secretion from human mesenchymal stromal cells. Stem Cell Res Ther, 2015. 6 : p. 164.

26. Dziki, J.L., et al., Solubilized extracellular matrix bioscaffolds derived from diverse source tissues differentially influence macrophage phenotype. J Biomed Mater Res A, 2017.105 (1): p. 138-147.

27. Badylak, S.F. and T.W. Gilbert, Immune response to biologic scaffold materials. Semin Immunol, 2008. 20 (2): p. 109-16.

28. Brown, B.N., et al., Macrophage polarization: an opportunity for improved outcomes in biomaterials and regenerative medicine.Biomaterials, 2012. 33 (15): p. 3792-802.

29. Brown, B.N., et al., Macrophage phenotype as a predictor of constructive remodeling following the implantation of biologically derived surgical mesh materials. Acta Biomater, 2012.8 (3): p. 978-87.

30. Fuertes, B., et al., Pacemaker lead displacement: mechanisms and management. Indian pacing and electrophysiology journal, 2003.3 (4): p. 231-238. 
31. Ip, J.E., L. Xu, and B.B. Lerman, Differences between cardiac implantable electronic device envelopes evaluated in an animal model. J Cardiovasc Electrophysiol, 2021. 32 (5): p. 1346-1354.

32. Mosser, D.M. and J.P. Edwards, Exploring the full spectrum of macrophage activation. Nat Rev Immunol, 2008. 8 (12): p. 958-69.

33. Wynn, T.A. and K.M. Vannella, Macrophages in Tissue Repair, Regeneration, and Fibrosis. Immunity, 2016. 44 (3): p. 450-462.

34. Sicari, B.M., et al., The promotion of a constructive macrophage phenotype by solubilized extracellular matrix. Biomaterials, 2014. 35 (30): p. 8605-12.

35. Shi, H.-X., et al., The Anti-Scar Effects of Basic Fibroblast Growth Factor on the Wound Repair In Vitro and In Vivo. PLOS ONE, 2013.8 (4): p. e59966.

36. Mosala Nezhad, Z., et al., Small intestinal submucosa extracellular matrix (CorMatrix@) in cardiovascular surgery: a systematic review. Interactive cardiovascular and thoracic surgery, 2016. 22 (6): p. 839-850.

37. Maxson, S., et al., Concise review: role of mesenchymal stem cells in wound repair. Stem Cells Transl Med, 2012. 1 (2): p. 142-9.

38. Voytik-Harbin, S.L., et al., Identification of extractable growth factors from small intestinal submucosa. J Cell Biochem, 1997.67 (4): p. 478-91.

39. Londono, R. and S.F. Badylak, Biologic scaffolds for regenerative medicine: mechanisms of in vivo remodeling. Ann Biomed Eng, 2015. 43 (3): p. 577-92.

40. Reing, J.E., et al., Degradation products of extracellular matrix affect cell migration and proliferation. Tissue Eng Part A, 2009. 15 (3): p. 605-14.

41. Boyd, W.D., et al., Pericardial reconstruction using an extracellular matrix implant correlates with reduced risk of postoperative atrial fibrillation in coronary artery bypass surgery patients. Heart Surg Forum, 2010. 13 (5): p. E311-6.

42. Stelly, M. and T.C. Stelly, Histology of CorMatrix Bioscaffold 5 Years After Pericardial Closure. The Annals of Thoracic Surgery, 2013. 96 (5): p. e127-e129.

43. Velnar, T., T. Bailey, and V. Smrkolj, The wound healing process: an overview of the cellular and molecular mechanisms. J Int Med Res, 2009. 37 (5): p. 1528-42.

44. Badylak, S.F., Decellularized allogeneic and xenogeneic tissue as a bioscaffold for regenerative medicine: factors that influence the host response. Ann Biomed Eng, 2014. 42 (7): p. 1517-27.

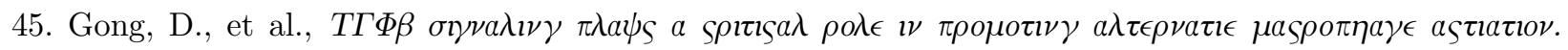
BMC Immunology, 2012.13 (1): p. 31. 